\title{
A case of osimertinib-resistant lung adenocarcinoma responded effectively to alternating therapy
}

\author{
Haruki Hirakawa ${ }^{1}$, Kazutoshi Komiya ${ }^{1}$, Chiho Nakashima ${ }^{1}$, Shinske Ogusu ${ }^{1}$, Tomomi Nakamura ${ }^{1}$, \\ Masahide Tanaka ${ }^{1}$, Koichiro Takahashi ${ }^{1}$, Yoshiaki Egashira ${ }^{2}$, Keita Kai ${ }^{3}$, Shinya Kimura ${ }^{1}$, Naoko \\ Sueoka-Aragane ${ }^{1}$
}

${ }^{1}$ Division of Hematology, Respiratory Medicine and Oncology, Department of Internal Medicine, ${ }^{2}$ Department of Radiology, Faculty of Medicine, Saga University, Saga, Japan; ${ }^{3}$ Department of Pathology, Saga University Hospital, Saga, Japan

Correspondence to: Naoko Sueoka-Aragane, MD, PhD. Division of Hematology, Respiratory Medicine and Oncology, Department of Internal Medicine, Faculty of Medicine, Saga University, 5-1-1 Nabeshima, Saga 849-8501, Japan. Email: sueokan@cc.saga-u.ac.jp.

\begin{abstract}
We report a case of initial lung adenocarcinoma in which transformation to small cell lung carcinoma (SCLC) was observed after acquired resistance to the $3^{\text {rd }}$ generation epidermal growth factor receptor tyrosine kinase inhibitor (EGFR-TKI) osimertinib and alternating treatment between chemotherapy and osimertinib was effective. A 61-year-old woman with EGFR mutation positive stage IV lung adenocarcinoma was administered $1^{\text {st }}$ generation EGFR-TKI for 8 months as the first line therapy, then chemotherapy and $2^{\text {nd }}$ generation EGFR-TKI after progressive disease (PD). Four years after initial diagnosis, EGFR T790M was detected in a metastatic lesion of the right thoracic wall and osimertinib was prescribed. Although partial response (PR) was achieved, a new metastatic lesion appeared in the right pleurum near the diaphragm, in which SCLC characteristics were observed with elevation of pro-gastrin-releasing peptide (pro-GRP) at the time of PD under osimertinib. Osimertinib was discontinued and carboplatin plus irinotecan chemotherapy was chosen as the next treatment, leading to PR after 2 cycles. Subsequently, the right thoracic wall tumor harboring T790M and the right pleural tumor near the diaphragm showing transformation to SCLC exhibited opposite responses to therapy alternating between osimertinib and chemotherapy. It is concluded that extended disease control can be achieved by combining appropriate treatments according to the mechanisms of resistance inferred from precise genetic and pathological examination in real time.
\end{abstract}

Keywords: Alternating therapy; transformation to small cell lung carcinoma (SCLC); osimertinib-resistant; non-small cell lung cancer

Submitted Jun 22, 2018. Accepted for publication Nov 09, 2018.

doi: $10.21037 /$ atm.2018.11.25

View this article at: http://dx.doi.org/10.21037/atm.2018.11.25

\section{Introduction}

Transformation to small cell lung carcinoma (SCLC) is one mechanism of tumor resistance to osimertinib (1-5). Although treatment targeted on small cell carcinoma has been effective, improved prognosis has not been achieved $(6,7)$. In addition, due to tumor heterogeneity, the mechanism of resistance varies depending on the site of the tumor, and multiple resistance mechanisms can co-exist $(8,9)$, making treatment more difficult. We experienced a case in which transformation to SCLC was observed after acquired resistance to osimertinib. Chemotherapy was effective against sites that transformed to SCLC, whereas osimertinib was effective against sites harboring T790M mutation. This case is a useful example of how disease control can be maintained over a long period by alternating therapies. 

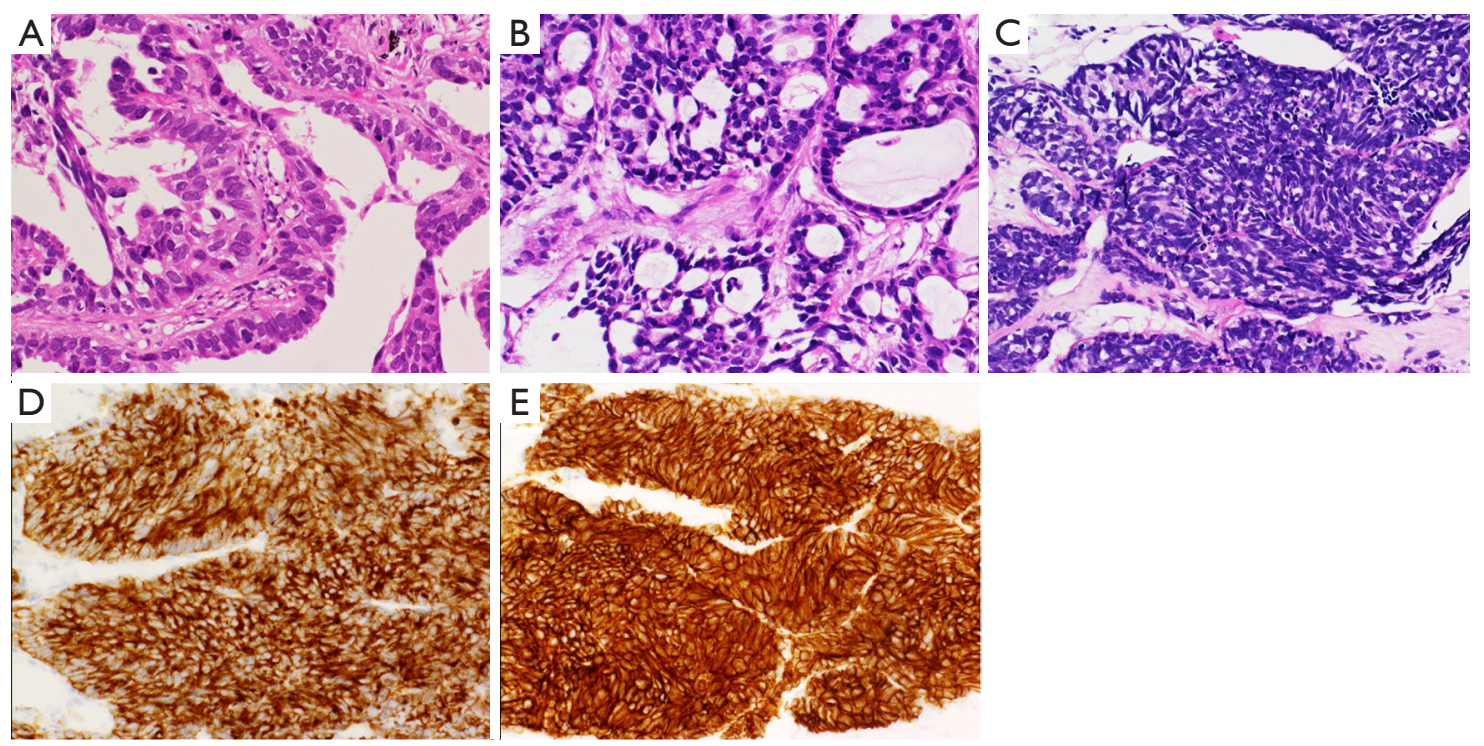

Figure 1 Pathology of initial biopsy and re-biopsy $\left(2^{\text {nd }}, 3^{\text {rd }}\right)$ tissue. (A) Papillary proliferation of malignant cells is observed in the biopsy specimen obtained from the primary tumor at the time of initial diagnosis (hematoxylin and eosin, $\times 200$ ); (B) re-biopsy specimen obtained from the metastatic lesion of the thoracic wall shows that malignant cells proliferated with formation of tubular structures (hematoxylin and eosin, $\times 200$ ); (C) third biopsy specimen obtained from the pleural tumor near the right diaphragm, which was resistant to osimertinib, shows dense proliferation of naked nucleoid malignant cells with highly increased chromatin (hematoxylin and eosin, $\times 200$ ). In immunohistological analysis, synaptophysin (D), and CD 56 (E) were both positive $(\times 200)$.

\section{Case presentation}

A 61-year-old woman with no smoking history presented with complaints of cough and sputum at her primary hospital. A tumor shadow was observed on the right upper lung field of a chest X-ray and she was referred to our hospital because of suspected lung cancer. Computed tomography (CT) showed a $40 \mathrm{~mm}$ mass in the upper lobe of the right lung, right pleural dissemination, and right pleural effusion. Magnetic resonance imaging (MRI) revealed multiple brain metastases. Positron emission tomography with computed tomography (PET/CT) showed intense uptake in the lung mass, pleural dissemination, and mediastinal lymph nodes. Serum levels of carcinoembryonic antigen (CEA) and sialyl Lewis X (SLX) tumor markers were high at $7.8 \mathrm{ng} / \mathrm{mL}$ and $22.6 \mathrm{U} / \mathrm{mL}$, but pro-gastrinreleasing peptide (pro-GRP) was within normal range at $25.0 \mathrm{pg} / \mathrm{mL}$. A CT guided biopsy from the primary lesion was performed and diagnosed as lung adenocarcinoma (Figure 1A), in which immunohistochemical stains were positive for thyroid transcription factor-1 (TTF-1) and negative for p63. In addition, Figure $1 B$ shows the second biopsy specimen and Figure 1C,D,E show the third biopsy specimen (details will be described later). Epidermal growth factor receptor $(E G F R)$ L858R was positive and stage was cT4N2M1b, IV (M: brain, pleural dissemination, UICC-7). Gefitinib was administered as the first line therapy, with a subsequent switch to erlotinib because of grade 3 aspartate aminotransferase (AST)/alanine aminotransferase (ALT) elevation; this resulted in partial response (PR). After 8 months without progression, chemotherapy with carboplatin, pemetrexed, and bevacizumab was given, followed by maintenance with pemetrexed and bevacizumab for 1.5 years. Subsequently, after 6 months of afatinib treatment the right pleural dissemination began to grow (Figure 2A).

Osimertinib was marketed 4.2 years after initial treatment of this patient, so at that time re-biopsy was performed from the metastatic lesion in the right chest wall. Proliferation of malignant cells showing tubular structures was observed (Figure 1B); these were TTF-1 positive and p40 negative. Since T790M was identified by the cobas EGFR mutation test v2 (Roche Molecular Diagnostics, Pleasanton, CA, USA) in addition to L858R, osimertinib was administered. The overall response was assessed as $\mathrm{PR}$, and disease control was maintained for approximately 10 months (Figure $2 A, B, C, D$ ), during which time there was 

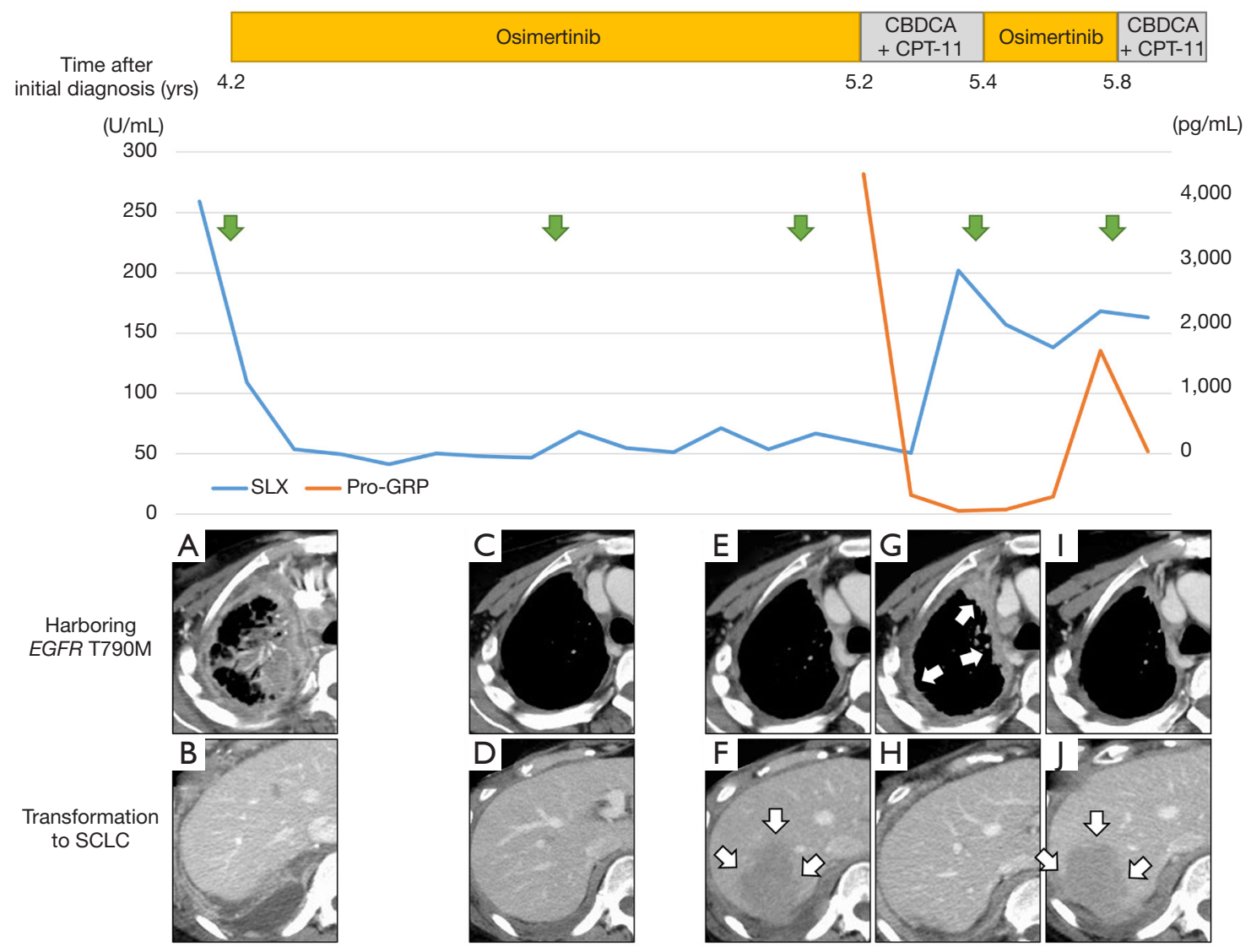

Figure 2 Clinical course from the administration of osimertinib is shown. Green arrows indicate points at which the CT examinations were performed. The upper part of the CT images (A,C,E,G,I) shows change of the right pleural dissemination and the lower part (B,D,F,H,J) shows change of the pleural tumor near the right diaphragm. SLX, sialyl Lewis X; pro-GRP, pro-gastrin-releasing peptide; SCLC, small cell lung carcinoma; CT, computed tomography.

no increase in brain metastases. Although shrinkage of the pleural dissemination within the right thorax was achieved (Figure 2E), the right pleural effusion gradually increased in size, and there was also a rapid increase in the pleural tumor near the right diaphragm (Figure 2F). The patient was hospitalized due to exacerbation of dyspnea and right abdominal pain; shortly after hospitalization, re-biopsy (third biopsy) was performed from the pleural tumor near the right diaphragm and transformation to SCLC was confirmed (Figure 1C). In immunohistochemical staining, synaptophysin (Figure 1D) and CD56 (Figure 1E) were positive as well as TTF-1. T790M and C797S were not detected in the tissue but L858R was. Features of small cell carcinoma were also observed in cytological examination of the right pleural effusion, co-existing with T790M. Tumor marker pro-GRP was high: 4,229 pg/mL. After 2 cycles of combination chemotherapy with carboplatin (CBDCA) and irinotecan (CPT-11) were conducted, pleural effusion decreased and the pleural tumor near the right diaphragm shrank markedly, and pro-GRP was within normal range. Dyspnea and right abdominal pain were also improved gradually within two weeks after starting chemotherapy. However, pleural dissemination within the right thorax once again progressed (Figure 2G,H), and SLX which decreased once after administration of osimertinib, increased again after two cycles of chemotherapy (Figure 2). Osimertinib was re-administered, and pleural dissemination within the right thorax again shrank (Figure 2I). On the other hand, the pleural tumor near the right diaphragm, which had shrunk under chemotherapy, again became enlarged (Figure 27). When treatment was changed from osimertinib to chemotherapy, pro-GRP declined. Alternating therapy between osimertinib and chemotherapy has continued for one year after occurrence of SCLC transformation. 


\section{Discussion}

Frequency of transformation to SCLC as a $1^{\text {st }}$ generation EGFR tyrosine kinase inhibitor (EGFR-TKI) resistance mechanism is reported in $2-14 \%$ of cases of EGFRmutated non-small cell lung cancer (10-13). Although the mechanism of acquired resistance to $3^{\text {rd }}$ generation EGFRTKI has not yet been elucidated, there are some reports of transformation to SCLC (1-5). In our case reported herein, the two lesions-one that transformed to SCLC and one that was T790M positive-grew differently depending on the treatment; the former at progressive disease (PD) to osimertinib and the latter at PD to chemotherapy. We are led to believe that the adenocarcinoma component harboring T790M, which was controlled by osimertinib, increased in size during administration of the cytotoxic agents. Because concurrent administration of EGFR-TKI and chemotherapy is not covered by medical insurance in Japan, we alternated therapy between osimertinib and chemotherapy, with the result that the disease condition is still stable. As far as we know, this is the first case where lesions with both adenocarcinoma and transformed SCLC have been controlled for a long period by alternating therapy.

There are several case reports showing that chemotherapy for SCLC was successful against transformation to SCLC after acquired resistant to $1^{\text {st }}$ generation EGFR-TKI. However, in two reviews, response rates were good but prognosis was poor $(6,7)$. Jiang et al. reported that the median survival time after confirmed transformation to SCLC was 7.1 months (6) and Roca et al. reported that it was 6.0 months (7). One of the causes of poor prognosis despite the good response to chemotherapy is multiple resistance mechanisms, which include tumor heterogeneity. Two types of tumor heterogeneity have been reported in terms of T790M and transformation to SCLC as mechanisms of acquired resistance to $1^{\text {st }}$ generation EGFRTKI. One is that T790M positive adenocarcinoma and transformation to SCLC reciprocally exist in multiple metastatic lesions, which were shown in autopsies of patients whose tumors had acquired resistance to $1^{\text {st }}$ generation EGFR-TKI $(8,9)$. The other is that histological change from adenocarcinoma to SCLC occurs in the same lesion after it has acquired resistance to $1^{\text {st }}$ generation EGFR-TKI (14). For both situations, combination of $3^{\text {rd }}$ generation EGFR-TKI and chemotherapy against SCLC would theoretically be effective. Although the use of a combination of osimertinib and cytotoxic agents has not been reported, it has been reported that disease control was achieved with a combination of alectinib and CPT-11 in a case of ALK-positive non-small cell lung cancer that had transformed to SCLC during alectinib treatment (15).

We performed alternating therapy with chemotherapy and osimertinib for a patient in whom transformation to SCLC was observed when the tumor acquired resistance to osimertinib. Considering this case, we think that it is important to perform genetic and pathological examination to precisely characterize the lesions in real time. A combination strategy, such as molecular targeted therapy and cytotoxic agents, will be required, since multiple resistance mechanisms arise in response to multiple treatments.

\section{Conclusions}

In addition to transformation to SCLC, various other mechanisms of acquired resistance to osimertinib have been reported. Due to the presence of tumor heterogeneity, it is conceivable that multiple resistance mechanisms may be present simultaneously or sequentially in an individual patient. Real time genetic and pathological examination to precisely characterize the lesions is indispensable for deciding the next course of treatment.

\section{Acknowledgements}

None.

\section{Footnote}

Conflicts of Interest: The authors have no conflicts of interest to declare.

Informed Consent: The patient gave informed consent for the use of biopsy specimens.

\section{References}

1. Piotrowska Z, Niederst MJ, Karlovich CA, et al. Heterogeneity Underlies the Emergence of EGFRT790 Wild-Type Clones Following Treatment of T790MPositive Cancers with a Third-Generation EGFR Inhibitor. Cancer Discov 2015;5:713-22.

2. Minari R, Bordi P, Del Re M, et al. Primary resistance to osimertinib due to SCLC transformation: Issue of T790M determination on liquid re-biopsy. Lung Cancer 
2018;115:21-7.

3. Ham JS, Kim S, Kim HK, et al. Two Cases of Small Cell Lung Cancer Transformation from EGFR Mutant Adenocarcinoma During AZD9291 Treatment. J Thorac Oncol 2016;11:e1-4.

4. Minari R, Bordi P, Tiseo M. Third-generation epidermal growth factor receptor-tyrosine kinase inhibitors in T790M-positive non-small cell lung cancer: review on emerged mechanisms of resistance. Transl Lung Cancer Res 2016;5:695-708.

5. Kim TM, Song A, Kim DW, et al. Mechanisms of Acquired Resistance to AZD9291: A MutationSelective, Irreversible EGFR Inhibitor. J Thorac Oncol 2015;10:1736-44.

6. Jiang SY, Zhao J, Wang MZ, et al. Small-Cell Lung Cancer Transformation in Patients With Pulmonary Adenocarcinoma: A Case Report and Review of Literature. Medicine (Baltimore) 2016;95:e2752.

7. Roca E, Gurizzan C, Amoroso V, et al. Outcome of patients with lung adenocarcinoma with transformation to small-cell lung cancer following tyrosine kinase inhibitors treatment: A systematic review and pooled analysis. Cancer Treat Rev. 2017;59:117-22.

8. Furugen M, Uechi K, Hirai J, et al. An Autopsy Case of Two Distinct, Acquired Drug Resistance Mechanisms in Epidermal Growth Factor Receptor-mutant Lung Adenocarcinoma: Small Cell Carcinoma Transformation

Cite this article as: Hirakawa $\mathrm{H}$, Komiya K, Nakashima C, Ogusu S, Nakamura T, Tanaka M, Takahashi K, Egashira Y, Kai K, Kimura S, Sueoka-Aragane N. A case of osimertinibresistant lung adenocarcinoma responded effectively to alternating therapy. Ann Transl Med 2018;6(23):464. doi: 10.21037/atm.2018.11.25 and Epidermal Growth Factor Receptor T790M Mutation. Intern Med 2015;54:2491-6.

9. Suda K, Murakami I, Sakai K, et al. Small cell lung cancer transformation and T790M mutation: complimentary roles in acquired resistance to kinase inhibitors in lung cancer. Sci Rep 2015;5:14447.

10. Kuiper JL, Heideman DA, Thunnissen E, et al. Incidence of T790M mutation in (sequential) rebiopsies in EGFRmutated NSCLC-patients. Lung Cancer 2014;85:19-24.

11. Tatematsu A, Shimizu J, Murakami Y, et al. Epidermal growth factor receptor mutations in small cell lung cancer. Clin Cancer Res 2008;14:6092-6.

12. Yu HA, Arcila ME, Rekhtman N, et al. Analysis of tumor specimens at the time of acquired resistance to EGFR-TKI therapy in 155 patients with EGFR-mutant lung cancers. Clin Cancer Res 2013;19:2240-7.

13. Sequist LV, Waltman BA, Dias-Santagata D, et al. Genotypic and histological evolution of lung cancers acquiring resistance to EGFR inhibitors. Sci Transl Med 2011;3:75ra26.

14. Morinaga R, Okamoto I, Furuta K, et al. Sequential occurrence of non-small cell and small cell lung cancer with the same EGFR mutation. Lung Cancer 2007;58:411-3.

15. Fujita S, Masago K, Katakami N, et al. Transformation to SCLC after Treatment with the ALK Inhibitor Alectinib. J Thorac Oncol 2016;11:e67-72. 